\title{
EDS-Lite, Quantitative Energy Dispersive Spectroscopy of Light Elements
}

\author{
Var L. St. Jeor \\ Cargill Incorporated; Dept. of Ingredient, Material, Nutrition, Minneapolis Research \& \\ Development Center, $1480028^{\text {th }}$ Avenue N, Plymouth, MN, USA 55447
}

Energy Dispersive Spectroscopy (EDS) is a tool of choice for many food research, food forensics and problem solving projects. Although the need for medium and heavy element analysis is common in foods, and includes EDS, ICP and XRF, the need for light element analysis should be obvious in the prevalence of Carbon, Nitrogen and Oxygen. These light elements can present some of our most unique challenges.

Nitrogen, for example, can be difficult to test for, and quantify. Existing thermal-based method such as Dumas, LECO or Kjeldahl (1830's technology ${ }^{1}$ ), are industry standards ${ }^{1}$, but are suspected of not detecting all chemical forms of Nitrogen ${ }^{2}$. Compensation factors are needed when calculating protein content with thermal-based methods. These compensation factors are unique from material-to-material (Corn vs. Soy for example). Typical ICP methods work in air, making nitrogen testing unlikely. So, the food industry is screaming for better technology.

When quantifying elements accurately, so-called standard-less ZAF methods are not reliable. ZAF methodology can be improved by using real standards. However, in our applications for light elements, a Least Squares method, using well qualified standards, is the most accurate and reproducible quantification technique we use. We have compared our EDS results against amino acid standards that have exacting known elemental compositions. Our techniques reproduce the chemical formula. We have also compared our results against the known thermal-basted methods and have found those thermal-based methods wanting.

Detecting most light elements, including nitrogen, is not difficult for EDS, but quantifying light elements accurately is another issue. Special considerations for instrument set-up are necessary when working with light elements, including lower acceleration voltages ${ }^{3,4}$. In many cases, a press is used to create a wafer of the sample material that is very smooth-surfaced. This eliminates $\mathrm{x}$-ray shadowing and improves the detected signal. Since foods are notorious for being asymmetrical in chemical and elemental make-up, multiple tests improve quantitation accuracy.

Light element EDS also allows us to image where these light elements are within the sample. For example, even though everything in a given sample may be carbon and oxygen, different food ingredients have different ratios of carbon and oxygen. When those ratios are sufficiently different, we can image oxygen-rich vs. carbon-rich regions uniquely, a form of chemical imaging by way of elemental content. 
Light element EDS analysis, using light element techniques, improve our analyses, making EDS a highly useful tool in food applications.

\section{References:}

[1] Williams, Phil; et. al.; Protein Testing Methods, Proceedings of the wheat protein symposium, pp. 37-47, 1998.

[2] Mike Porter, personal communication, Cargill, Nov. 2009.

[3] Echlin, P; Low Voltage Energy Dispersive Microanalysis of Bulk Organic Materials, Microscopy \& Microanalysis, 5, suppl. 2 proceedings, pp.312-313, 1999.

[4] Joy, D.C.; The Efficiency of X-ray Production at Low Energies, J. Microsc, 191, pp.74-82, 1998.

Figure 1, Left, is a plot of nitrogen concentrations for various amino acids, and other nitrogencontaining materials, using low voltage methods. L-Proline was used as our standard. Blue is the nitrogen concentration based upon the chemical formulas. Pink is EDS-stated nitrogen concentrations using low voltage, standard-less ZAF quantification methods. Right, the same data, but with the EDS technique quantified using a least-squares method. Urea is out of reach of our standard, Erucamide nitrogen was present in quantities too low for quantification. The other substances are well within the reach of our standard.

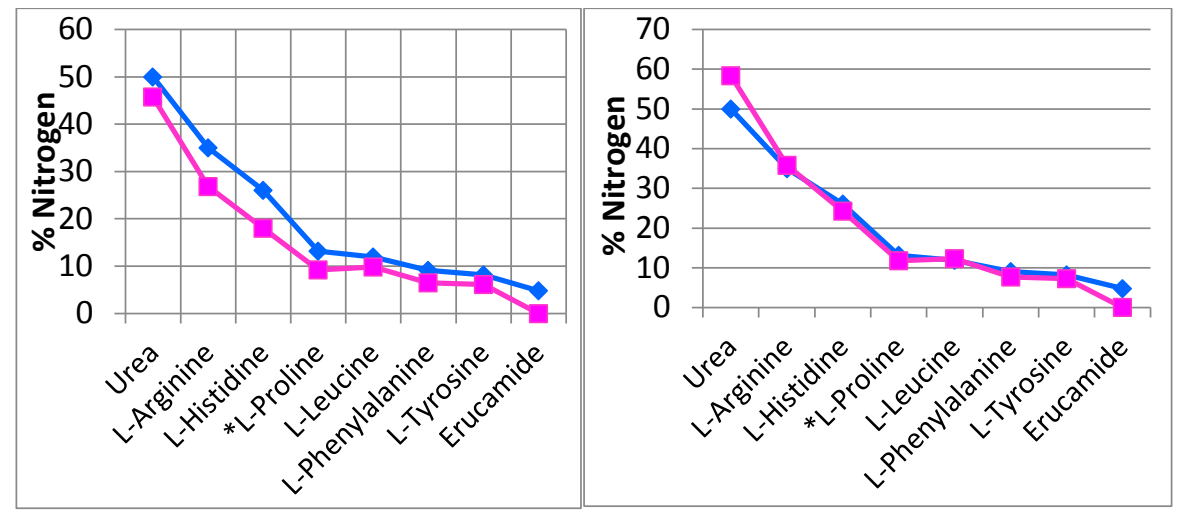

Figure 2. EDS elemental mapping being use as a form of chemical imaging. An EDS dot map of Erithritol crystals (yellow) with another non-sugar sweetener (pink) resting on the crystal surfaces. EDS uses differing carbon:oxygen ratios to distinguish the two sweeteners.

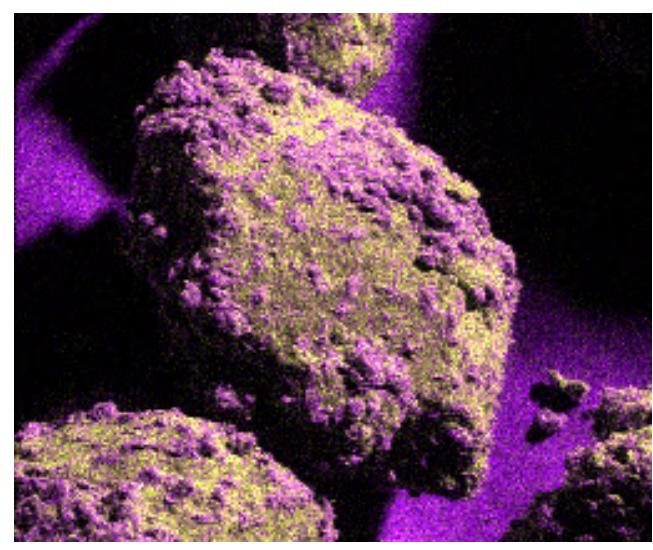

